\title{
Evaluation of 5-HTTLPR (insertion/deletion) and BDNF (rs6265) genetic variations in the Slovakian individuals suffering from affective disorders
}

\author{
Aneta Bednarova ${ }^{1,2, \star}$, Martina Cizmarikova ${ }^{3, *}$, Viera Habalova $^{4}$ and Dominika Jarcuskova ${ }^{2,5}$ \\ ${ }^{1} 2^{\text {nd }}$ Department of Psychiatry, Faculty of Medicine, Pavol Jozef Safarik University in Kosice, Kosice, Slovakia \\ ${ }^{2}$ Louis Pasteur University Hospital, Kosice, Slovakia \\ ${ }^{3}$ Department of Pharmacology, Faculty of Medicine, Pavol Jozef Safarik University in Kosice, Kosice, Slovakia \\ ${ }^{4}$ Department of Medical Biology, Faculty of Medicine, Pavol Jozef Safarik University in Kosice, Kosice, Slovakia \\ ${ }^{5} 1^{\text {st }}$ Department of Psychiatry, Faculty of Medicine, Pavol Jozef Safarik University in Kosice, Kosice, Slovakia
}

\begin{abstract}
The pathophysiology of affective disorders (AD), including depressive disorders (DD) and anxiety disorders (ANXD), is still unclear. To understand risk factors of the disorders, we evaluated genetic variations of the serotonin reuptake transporter (5-HTTLPR, ins/del) and the brain-derived neurotrophic factor $(B D N F$, rs6265) in Slovak patients suffering from AD. After genotyping we observed a significantly increased frequency of LS and LL genotypes (5-HTTLPR) in individuals diagnosed with $\mathrm{AD}$ compared to controls $(\mathrm{OR}=1.99,95 \% \mathrm{CI}=1.21-3.27, p=0.006)$. There was also a significant relationship between $\mathrm{TT}(B D N F)$ genotype and the risk of $\mathrm{AD}$ in males $(\mathrm{OR}=5.93$, 95\% CI $=1.42-27.07, p=0.011)$. In gene-gene analysis, the LL or LS (5-HTTLPR) and CT or TT $(B D N F)$ genotype combinations had a risk-enhancing effect on AD susceptibility (mainly ANXD in males), while SS (5-HTTLPR) and TT (BDNF) combination had a protective effect on AD risk (mainly ANXD). However, larger prospective studies are needed to confirm our findings.
\end{abstract}

Key words: 5-HTTLPR - BDNF - Affective disorder - Depressive disorder - Anxiety disorder

Abbreviations: AD, affective disorder; ANXD, anxiety disorder; BD, bipolar depression; BDNF, brain-derived neurotrophic factor; DD, depressive disorder; GAD, generalized anxiety disorder; 5-HT, serotonin; 5-HTTLPR, genetic variation of the serotonin reuptake transporter; HWE, Hardy-Weinberg equilibrium; OCD, obsessive-compulsive disorder; PTSD, post-traumatic stress disorder; SLC6A4, gene encoded serotonin reuptake transporter; SERT, serotonin reuptake transporter; UD, unipolar depression.

\section{Introduction}

Affective disorders (ADs) are serious, mostly recurrent, or chronic mental disorders that affect the general emotional state of a patient. Although they are not clearly defined diagnostic entities, according to the International Society for

\footnotetext{
* These authors contributed equally to this work.

Correspondence to: Martina Cizmarikova, Department of Pharmacology, Faculty of Medicine, Pavol Jozef Safarik University in Kosice, Trieda SNP 1, 04011 Kosice, Slovakia

E-mail: martina.cizmarikova@upjs.sk
}

Affective Disorders (ISAD, 2021, https://www.isad.org.uk/), they include unipolar depression (UD) and bipolar depression (BD), generalized anxiety disorder (GAD), and more specific anxiety disorders, such as social phobia, agoraphobia, panic disorder, obsessive-compulsive disorder (OCD) and post-traumatic stress disorder (PTSD). Moreover, depressive and anxiety conditions can occur simultaneously in the same patient. Generally, the ISAD recommends considering all these disorders as a single group because of a high level of similarity between them. It is estimated that over $4.4 \%$ of the world's population suffers from any type of depressive disorders (DDs) and 3.6\% of the global population from

(c) The Authors 2021. This is an open access article under the terms of the Creative Commons Attribution-NonCommercial 4.0 International License (https://creativecommons.org/licenses/by-nc/4.0/), which permits non-commercial use, distribution, and reproduction in any medium, provided the original work is properly cited. 
anxiety disorders (ANXDs) (World Health Organization 2017). On the other hand, according to the International Statistical Classification of Diseases and Related Health Problems (ICD), usually only unipolar affective disorder and bipolar affective disorder are considered as ADs.

The precise pathophysiology of ADs is still unclear despite a substantial research effort in this field. Thus, many potential biomarkers for $\mathrm{AD}$ risk, as well as genetic variations of such molecules are studied as possible causal factors of the disorder.

The hypothesis of serotonin (5-HT) dysregulation in the development of depression and anxiety has been attracting attention for several decades (Liu et al. 2018). There has also been a growing interest in the study of the serotonin reuptake transporter (SERT) which is considered the main molecular target of selective serotonin reuptake inhibitors, the most widely utilized antidepressants. This transporter, also known as solute carrier family 6 , member 4 , is encoded by the SLC6A4 gene located on chromosome 17. Concerning ADs, considerable attention has been mainly paid to its functional 5-HTTLPR genetic variation that represents the insertion (ins) generating the long ("L") allele or deletion (del) generating the short ("S") allele in the promoter region of the gene (Iurescia et al. 2016). The data appear to suggest that the $S$ allele variant can result in reduced transcriptional efficiency of the promoter, leading to lower availability and decreased reuptake activity of the transporter in comparison with L allele (Lesch et al. 1996). Many studies monitoring the role of ins/del genetic variation in ADs risk have been published, however, with inconsistent results (Mohamed Saini et al. 2012; Jiménez et al. 2019; Miozzo et al. 2020).

Much attention is also paid to the brain-derived neurotrophic factor (BDNF). This protein, widely expressed in the central nervous system, is involved in the control of neuronal growth, survival, neurogenesis, and differentiation, neurotransmitter signalling, and synaptic plasticity (Park and Poo 2013). Several publications indicate that its dysregulation might have a role in the etiology of diverse neuropsychiatric conditions, including DDs and ANXDs (Lin and Huang 2020). For instance, lower plasma and serum BDNF levels were found in patients with depression than in controls (BocchioChiavetto et al. 2010). Similarly, much research on the most common single nucleotide $B D N F$ genetic variation $196 \mathrm{C}>\mathrm{T}$ ( $\mathrm{rs6265;}$ MAF $=0.20)$ in exon 5 located on chromosome 11p13 has been done. This genetic variation results in an amino acid substitution of valine (Val) for methionine (Met) at codon 66 (Val66Met). Although the Met allele was associated with decreased cellular trafficking, processing, and activity-dependent BDNF secretion, impairment of synaptic transmission, and cortical plasticity, as well as with changes in an extracellular level of BDNF (Egan et al. 2003; Pattwell et al. 2012), its role in AD susceptibility is controversial (Verhagen et al. 2010; Gyekis et al. 2013; Zhao et al. 2018; Border et al. 2019).
Although some data indicated an interaction between BDNF and serotonin signalling in mood control (Martinowich and Lu 2008), only several studies were aimed at the evaluation of gene-gene interaction of the described genetic variations with AD susceptibility (Grabe et al. 2012; Kostic et al. 2016; Nestor et al. 2019; Wang et al. 2020).

Based on the inconsistency of data published earlier, our study focused on determining the relevance of the 5-HT$T L P R$ (ins/del) and BDNF (Val66Met), including their genegene interactions, to the risk of development of ADs in the population sample of Slovakian individuals.

\section{Material and Methods}

\section{Participants}

The current case-control study was performed in two groups of adult participants of Slovak origin (Caucasians). The case (patient) group comprised individuals aged 18 and above at the time of onset of any type of $\mathrm{AD}$ who were treated or monitored at the $2^{\text {nd }}$ Department of Psychiatry of Pavol Jozef Safarik University in Kosice, Slovakia. All patients were on antidepressant treatment in the acute phase of the disorder.

Exclusion criteria included: organic mental disorders, mental retardation, personality disorders, drug or alcohol abuse, psychotic symptoms, suicidal behavior, and pregnancy.

The control group with adult volunteers had no relation to the cases and was without any known psychiatric disorder.

Blood samples from both groups were collected from November 2018 to January 2021 and participation in testing was voluntary and could be cancelled by any individual at any time during the study.

The clinical diagnosis was made or confirmed by two certified psychiatrists who were kept blind to the diagnosis made by one to another and sample genotyping.

This study was approved by the local ethics committee, and all subjects provided written informed consent.

\section{Genotyping}

DNA from the whole blood was extracted with a Wizard Genomic DNA Isolation kit (Promega, Co, Ltd, USA). Genotyping of the BDNF rs6265 was performed using asymmetric real-time polymerase chain reaction and subsequent high-resolution melting analysis in the presence of an unlabelled probe on the Eco Real-Time PCR System (Illumina, Inc., San Diego, CA, USA). The oligonucleotides (forward limit: 5'-GCCGAACTTTCTGGTCCTCATCC-3', reverse excess: 5'-AAGGCAGGTTCAAGAGGCTTG-3', and probe: 5'-GCTCTTCTATCACGTGTTCGAAAGTGTC-Phos) were designed in our laboratory. Genotypes were identified using Eco ${ }^{\mathrm{TM}}$ Software 4.1. 
The 5-HTTLPR genetic variation was genotyped using forward 5'-GGCGTTGCCGCTCTGAATGC-3' and reverse 5'-GAGGGACTGAGCTGGACAACCAC-3' primers according to Murakami et al. (1999). The PCR products were separated by electrophoresis on a $2 \%$ agarose gel.

\section{Statistical analysis}

SPSS software for Windows (version 16.0, USA) and GraphPad Prism 9 (GraphPad Software, Inc., USA) were used for all statistical analyses. A $p$-value of $<0.05$ was taken as statistically significant. The Chi-square or Fisher's exact tests were conducted to compare contingency tables, while the strength of the relative associations was assessed via odds ratios (ORs) with their corresponding 95\% confidence intervals (CIs). Quantitative values were evaluated using the Mann-Whitney U test. The Hardy-Weinberg equilibrium (HWE) assumption was assessed for the tested groups by comparing the observed numbers of each genotype with those expected under the HWE for the estimated allele frequency. Codominant, dominant, recessive, and overdominant genetic models were used to analyze the association between a genetic variation and phenotype. Likewise, we used binary logistic regression analysis to determine the role of tested genetic variations as independent risk factors for affective disorder susceptibility.

\section{Results}

\section{Characteristics of the participants}

A total of 500 subjects were included in the current study. Out of 280 patients, 183 (65.4\%) were females and 97 (34.6\%) were males (female:male ratio: 1.9:1). The mean age of the cases was $50.3 \pm 13.9$ years (range: $18-83$ years).

Overall, $222(79.3 \%)$ patients suffered from depressive disorders - DDs (UD: 142 females and 62 males; BD: 9 females and 9 males), and 58 (20.7\%) patients were diagnosed with anxiety disorders - ANXDs (GAD: 26 females and 18 males; OCD: 4 females and 4 males; panic disorder and social phobia: 1 male; PTSD: 2 females and 3 males).

Age-, gender-, and ethnicity-matched healthy adults served as the control group. Out of 220 individuals, 134 (60.9\%) were females and $86(39.1 \%)$ were males.

All collected blood samples were successfully genotyped for both genetic variations. The relevant genotype and allele frequencies of patients and controls are shown

Table 1. Genotype and allele frequencies of 5-HTTLPR (ins/del) and BDNF (Val66Met, rs6265)

\begin{tabular}{|c|c|c|c|c|c|c|c|c|c|c|}
\hline \multirow{3}{*}{ Variable } & \multicolumn{5}{|c|}{ 5-HTTLPR (ins/del) } & \multicolumn{5}{|c|}{$B D N F($ rs6265) } \\
\hline & \multicolumn{3}{|c|}{ Genotypes } & \multicolumn{2}{|c|}{ Alleles } & \multicolumn{3}{|c|}{ Genotypes } & \multicolumn{2}{|c|}{ Alleles } \\
\hline & LL (\%) & LS (\%) & SS (\%) & $\mathrm{L}(\%)$ & $\mathrm{S}(\%)$ & CC (\%) & CT (\%) & $\mathrm{TT}(\%)$ & $\mathrm{C}(\%)$ & $\mathrm{T}(\%)$ \\
\hline \multicolumn{11}{|l|}{ Controls } \\
\hline Total & $68(30.9)$ & $107(48.6)$ & $45(20.5)$ & $243(55.2)$ & $197(44.8)$ & $144(65.5)$ & $64(29.1)$ & $12(5.4)$ & $352(80.0)$ & $88(20.0)$ \\
\hline Females & $46(34.3)$ & $64(47.8)$ & $24(17.9)$ & $156(58.2)$ & $112(41.8)$ & $82(61.2)$ & $42(31.3)$ & $10(7.5)$ & $206(76.9)$ & $62(23.1)$ \\
\hline Males & $22(25.6)$ & $43(50.0)$ & $21(24.4)$ & $87(50.6)$ & $85(49.4)$ & $62(72.1)$ & $22(25.6)$ & $2(2.3)$ & $146(84.9)$ & $26(15.1)$ \\
\hline \multicolumn{11}{|l|}{$\mathrm{AD}$} \\
\hline Total & $102(36.4)$ & $146(52.1)$ & $32(11.4)$ & $350(62.5)$ & $210(37.5)$ & $178(63.6)$ & $78(27.9)$ & $24(8.6)$ & $434(77.5)$ & $126(22.5)$ \\
\hline Females & $72(39.3)$ & $90(49.2)$ & $21(11.5)$ & $234(63.9)$ & $132(36.1)$ & $124(67.8)$ & $47(25.7)$ & $12(6.6)$ & $295(80.6)$ & $71(19.4)$ \\
\hline Males & $30(30.9)$ & $56(57.7)$ & $11(11.3)$ & $116(59.8)$ & $78(40.2)$ & $54(55.7)$ & $31(32.0)$ & $12(12.4)$ & $139(71.6)$ & $55(28.4)$ \\
\hline \multicolumn{11}{|l|}{ DD } \\
\hline Total & $79(35.6)$ & $114(51.4)$ & $29(13.1)$ & $272(61.3)$ & $172(38.7)$ & $147(66.2)$ & $55(24.8)$ & $20(9.0)$ & $349(78.6)$ & 95 (21.4) \\
\hline Females & $59(39.1)$ & $74(49.0)$ & $18(11.9)$ & $192(63.6)$ & $110(36.4)$ & $104(68.9)$ & $36(23.8)$ & $11(7.3)$ & $244(80.8)$ & $58(19.2)$ \\
\hline Males & $20(28.2)$ & $40(56.3)$ & $11(15.5)$ & $80(56.3)$ & $62(43.7)$ & $43(60.6)$ & $19(26.8)$ & $9(12.7)$ & $105(73.9)$ & $37(26.1)$ \\
\hline \multicolumn{11}{|l|}{ UD } \\
\hline Total & $76(37.3)$ & $101(49.5)$ & $27(13.2)$ & $253(62.0)$ & $155(38.0)$ & $134(65.7)$ & $52(25.5)$ & $18(8.8)$ & $320(78.4)$ & 88 (21.6) \\
\hline Females & $56(39.4)$ & 68 (47.9) & $18(12.7)$ & $180(63.4)$ & $104(36.6)$ & $96(67.6)$ & $35(24.6)$ & $11(7.7)$ & $227(79.9)$ & $57(20.1)$ \\
\hline Males & $20(32.3)$ & $33(53.2)$ & $9(14.5)$ & $73(58.9)$ & $51(41.1)$ & $38(61.3)$ & $17(27.4)$ & $7(11.3)$ & $93(75.0)$ & $31(25.0)$ \\
\hline \multicolumn{11}{|l|}{ ANXD } \\
\hline Total & $23(39.7)$ & $32(55.2)$ & $3(5.2)$ & $78(67.2)$ & $38(32.8)$ & $31(53.4)$ & $23(39.7)$ & $4(6.9)$ & $85(73.3)$ & $31(26.7)$ \\
\hline Females & $13(40.6)$ & $16(50.0)$ & $3(9.4)$ & $42(65.6)$ & $22(34.4)$ & $20(62.5)$ & $11(34.4)$ & $1(3.1)$ & $51(79.7)$ & $13(20.3)$ \\
\hline Males & $10(38.5)$ & $16(61.5)$ & $0(0.0)$ & $36(69.2)$ & $16(30.8)$ & $11(42.3)$ & $12(46.2)$ & $3(11.5)$ & $34(65.4)$ & 18 (34.6) \\
\hline
\end{tabular}

$\mathrm{AD}$, affective disorder; $\mathrm{ANXD}$, anxiety disorder; $\mathrm{DD}$, depressive disorder; UD, unipolar depression. 
in Table 1 . The $\mathrm{L}(5-H T T L P R)$ and $\mathrm{C}(B D N F)$ alleles were found to be more frequent in screened groups of participants.

The genotype distribution among controls did not deviate from HWE $(p \geq 0.05)$ for both tested genetic variations. Similarly, the HWE was satisfactory in the observed genotype frequencies for the 5-HTTLPR in the patient group. However, the distribution of the BDNF genotypes among the cases showed a deviation from $\operatorname{HWE}(p<0.01)$.
Impact of 5-HTTLPR (ins/del) and BDNF (Val66Met) on the risk of $A D$

Firstly, we assessed genotype and allele distributions between all patients with ADs (involvement of both DDs and ANXDs, $n=280$ ) compared to control individuals.

We detected a statistically significant association between 5-HTTLPR and the disorder development in the codominant (LL vs. SS: reciprocal of $\mathrm{OR}=2.11,95 \% \mathrm{CI}=1.23-3.71, p=$

Table 2. Distribution of genotypes of 5-HTTLPR (ins/del) among cases (patients with AD) and controls

\begin{tabular}{|c|c|c|c|c|}
\hline Genotypes of 5-HTTLPR (ins/del) & Case $n(\%)$ & Control $n(\%)$ & OR (95\% CI) & $p$ value \\
\hline \multicolumn{5}{|l|}{$\mathrm{AD}$ - all patients } \\
\hline LL & $102(36.4)$ & $68(30.9)$ & $1.00^{\mathrm{a}}$ & \\
\hline LS & $146(52.1)$ & $107(48.6)$ & $0.91(0.61-1.37)$ & 0.639 \\
\hline \multirow[t]{2}{*}{ SS } & $32(11.4)$ & $45(20.5)$ & $0.47(0.27-0.81)$ & $0.007^{*, x} / 0.009^{*, \mathrm{f}}$ \\
\hline & & & $2.11(1.23-3.71)^{\mathrm{r}}$ & \\
\hline $\mathrm{LL}+\mathrm{LS}$ & $248(88.6)$ & $175(79.5)$ & $1.00^{\mathrm{c}}$ & \\
\hline \multirow[t]{2}{*}{ SS } & $32(11.4)$ & $45(20.5)$ & $0.50(0.31-0.83)$ & $0.006^{\star, x} / 0.006^{*, \mathrm{f}}$ \\
\hline & & & $1.99(1.21-3.27)^{\mathrm{r}}$ & \\
\hline \multicolumn{5}{|l|}{$\mathrm{AD}$ - males } \\
\hline LL & $30(30.9)$ & $22(25.6)$ & $1.00^{\mathrm{a}}$ & \\
\hline LS & $56(57.7)$ & $43(50.0)$ & $0.95(0.48-1.88)$ & 0.894 \\
\hline \multirow[t]{2}{*}{ SS } & $11(11.3)$ & $21(24.4)$ & $0.38(0.15-0.98)$ & $0.038^{\star, \chi} / 0.045^{\star, f}$ \\
\hline & & & $2.60(1.02-6.68)^{r}$ & \\
\hline $\mathrm{LL}+\mathrm{LS}$ & $86(88.7)$ & $65(75.6)$ & $1.00^{\mathrm{c}}$ & \\
\hline \multirow[t]{2}{*}{ SS } & $11(11.3)$ & $21(24.4)$ & $0.40(0.18-0.86)$ & $0.020^{\star, \chi} / 0.031^{\star, f}$ \\
\hline & & & $2.53(1.16-5.41)^{\mathrm{r}}$ & \\
\hline \multicolumn{5}{|l|}{ DD - all patients } \\
\hline LL & $79(35.6)$ & $68(30.9)$ & $1.00^{\mathrm{a}}$ & \\
\hline LS & $114(51.4)$ & $107(48.6)$ & $0.92(0.61-1.39)$ & 0.685 \\
\hline \multirow[t]{2}{*}{ SS } & $29(13.1)$ & $45(20.5)$ & $0.56(0.32-0.99)$ & $0.041^{\star, \chi} / 0.047^{\star, f}$ \\
\hline & & & $1.80(1.01-3.17)^{\mathrm{r}}$ & \\
\hline $\mathrm{LL}+\mathrm{LS}$ & $193(86.9)$ & $175(79.5)$ & $1.00^{\mathrm{c}}$ & \\
\hline \multirow[t]{2}{*}{ SS } & $29(13.1)$ & $45(20.5)$ & $0.58(0.35-0.96)$ & $0.037^{\star, x} / 0.042^{\star, \mathrm{f}}$ \\
\hline & & & $1.71(1.04-2.82)^{\mathrm{r}}$ & \\
\hline \multicolumn{5}{|l|}{ UD - all patients } \\
\hline LL & $76(37.3)$ & $68(30.9)$ & $1.00^{\mathrm{a}}$ & \\
\hline LS & $101(49.5)$ & $107(48.6)$ & $0.85(0.55-1.28)$ & 0.436 \\
\hline \multirow[t]{2}{*}{ SS } & $27(13.2)$ & $45(20.5)$ & $0.54(0.30-0.97)$ & $0.034^{\star, \chi} / 0.043^{\star, \mathrm{f}}$ \\
\hline & & & $1.86(1.03-3.36)^{\mathrm{r}}$ & \\
\hline \multicolumn{5}{|l|}{ ANXD - all patients } \\
\hline LL & $23(39.7)$ & $68(30.9)$ & $1.00^{\mathrm{a}}$ & \\
\hline LS & $32(55.2)$ & $107(48.6)$ & $0.88(0.47-1.64)$ & 0.695 \\
\hline \multirow[t]{2}{*}{ SS } & $3(5.2)$ & $45(20.5)$ & $0.20(0.06-0.65)$ & $0.006^{*, x} / 0.006^{*, \mathrm{f}}$ \\
\hline & & & $5.07(1.55-16.66)^{\mathrm{r}}$ & \\
\hline $\mathrm{LL}+\mathrm{LS}$ & $55(94.8)$ & $175(79.5)$ & $1.00^{\mathrm{c}}$ & \\
\hline \multirow[t]{2}{*}{ SS } & $3(5.2)$ & $45(20.5)$ & $0.21(0.07-0.65)$ & $0.006^{*, x} / 0.006^{*, f}$ \\
\hline & & & $4.71(1.53-14.93)^{\mathrm{r}}$ & \\
\hline
\end{tabular}

$\mathrm{AD}$, affective disorder; $\mathrm{ANXD}$, anxiety disorder; $\mathrm{CI}$, confidence interval; $\mathrm{DD}$, depressive disorder; OR, odds ratio; UD, unipolar depression; * significant association; ${ }^{\mathrm{a}}$ codominant model; ${ }^{\mathrm{c}}$ recessive model; ${ }^{\mathrm{f}}$ Fisher's exact test; ${ }^{\mathrm{r}}$ reciprocal of OR with the relevant CI; ${ }^{\mathrm{X}}$ Chi-square test. 
Table 3. Distribution of genotypes of BDNF (Val66Met, rs6265) among male cases (patients with AD) and controls

\begin{tabular}{|c|c|c|c|c|}
\hline Genotypes of BDNF (rs6265) & Case $n(\%)$ & Control $n(\%)$ & OR $(95 \% \mathrm{CI})$ & $p$ value \\
\hline \multicolumn{5}{|l|}{ AD - males } \\
\hline $\mathrm{CC}$ & $54(55.7)$ & $62(72.1)$ & $1.00^{\mathrm{a}}$ & \\
\hline $\mathrm{CT}$ & $31(32.0)$ & $22(25.6)$ & $1.61(0.83-3.03)$ & 0.150 \\
\hline $\mathrm{TT}$ & $12(12.4)$ & $2(2.3)$ & $6.89(1.58-31.64)$ & $0.006^{*, \mathrm{x}} / 0.009^{*, \mathrm{f}}$ \\
\hline $\mathrm{CC}$ & $54(55.7)$ & $62(72.1)$ & $1.00^{\mathrm{b}}$ & \\
\hline $\mathrm{CT}+\mathrm{TT}$ & $43(44.3)$ & $24(27.9)$ & $2.06(1.10-3.84)$ & $0.021^{\star, x} / 0.031^{\star, f}$ \\
\hline $\mathrm{CC}+\mathrm{CT}$ & $85(87.6)$ & $84(97.7)$ & $1.00^{\mathrm{c}}$ & \\
\hline $\mathrm{TT}$ & $12(12.4)$ & $2(2.3)$ & $5.93(1.42-27.07)$ & $0.011^{\star, \mathrm{X}} / 0.012^{\star, \mathrm{f}}$ \\
\hline \multicolumn{5}{|l|}{ DD - males } \\
\hline $\mathrm{CC}$ & $43(60.6)$ & $62(72.1)$ & $1.00^{\mathrm{a}}$ & \\
\hline $\mathrm{CT}$ & $19(26.8)$ & $22(25.6)$ & $1.25(0.62-2.50)$ & 0.554 \\
\hline $\mathrm{TT}$ & $9(12.7)$ & $2(2.3)$ & $6.49(1.54-30.69)$ & $0.010^{\star, \mathrm{X}} / 0.012^{\star, \mathrm{f}}$ \\
\hline $\mathrm{CC}+\mathrm{CT}$ & $62(87.3)$ & $84(97.7)$ & $1.00^{\mathrm{c}}$ & \\
\hline $\mathrm{TT}$ & $9(12.7)$ & $2(2.3)$ & $6.10(1.49-28.66)$ & $0.011^{\star, \mathrm{X}} / 0.024^{\star, \mathrm{f}}$ \\
\hline \multicolumn{5}{|l|}{ UD - males } \\
\hline $\mathrm{CC}$ & $38(61.3)$ & $62(72.1)$ & $1.00^{\mathrm{a}}$ & \\
\hline $\mathrm{CT}$ & $17(27.4)$ & $22(25.6)$ & $1.26(0.59-2.61)$ & 0.545 \\
\hline $\mathrm{TT}$ & $7(11.3)$ & $2(2.3)$ & $5.71(1.14-27.90)$ & $0.020^{\star, x} / 0.031^{\star, f}$ \\
\hline $\mathrm{CC}+\mathrm{CT}$ & $55(88.7)$ & $84(97.7)$ & $1.00^{\mathrm{c}}$ & \\
\hline $\mathrm{TT}$ & $7(11.3)$ & $2(2.3)$ & $5.35(1.11-25.94)$ & $0.024^{\star, x} / 0.035^{\star, f}$ \\
\hline \multicolumn{5}{|l|}{ ANXD - males } \\
\hline $\mathrm{CC}$ & $11(42.3)$ & $62(72.1)$ & $1.00^{\mathrm{a}}$ & \\
\hline $\mathrm{CT}$ & $12(46.2)$ & $22(25.6)$ & $3.07(1.18-8.30)$ & $0.018^{\star, x} / 0.024^{\star, f}$ \\
\hline $\mathrm{TT}$ & $3(11.5)$ & $2(2.3)$ & $8.46(1.52-49.68)$ & $0.011^{\star, \mathrm{X}} / 0.038^{\star, \mathrm{f}}$ \\
\hline $\mathrm{CC}$ & $11(42.3)$ & $62(72.1)$ & $1.00^{\mathrm{b}}$ & \\
\hline $\mathrm{CT}+\mathrm{TT}$ & $15(57.7)$ & $24(27.9)$ & $3.52(1.36-8.87)$ & $0.005^{\star, x} / 0.009^{\star, \mathrm{f}}$ \\
\hline
\end{tabular}

$\mathrm{AD}$, affective disorder; $\mathrm{ANXD}$, anxiety disorder; CI, confidence interval; $\mathrm{DD}$, depressive disorder; OR, odds ratio; UD, unipolar depression; ${ }^{*}$ significant association; ${ }^{\mathrm{a}}$ codominant model; ${ }^{\mathrm{b}}$ dominant model; ${ }^{\mathrm{c}}$ recessive model; ${ }^{\mathrm{f}}$ Fisher's exact test; ${ }^{\mathrm{X}}$ Chi-square test.

$0.007)$ and recessive (LL+LS vs. SS: reciprocal of $\mathrm{OR}=1.99$, 95\% CI $=1.21-3.27, p=0.006$ ) models (Table 2 ). In brief, individuals carrying at least one $\mathrm{L}$ allele had a significantly higher risk of $\mathrm{AD}$ development. The difference in allele frequencies between patients suffering from $\mathrm{AD}$ and controls was also found to be statistically significant (L vs. S: OR = $1.35,95 \% \mathrm{CI}=1.05-1.74, p=0.020)$. No differences in $B D N F$ genotype distributions were observed between cases and controls.

Secondly, the comparative analyses were gender-stratified. The significant values were observed only in male samples. The difference in genotype frequencies of the 5-HTTLPR between controls and cases was found to be statistically significant in the codominant (LL $v$ s. SS: reciprocal of OR = $2.60,95 \% \mathrm{CI}=1.02-6.68, p=0.038)$ and recessive $(\mathrm{LL}+\mathrm{LS} v s$. SS: reciprocal of OR $=2.53,95 \% \mathrm{CI}=1.16-5.41, p=0.020$ ) models (Table 2).

A significant link between $\mathrm{AD}$ susceptibility in males and $B D N F$ genotypes in the codominant (TT vs. CC: $\mathrm{OR}=6.89$,
95\% CI $=1.58-31.64, p=0.009)$, dominant $(\mathrm{CT}+\mathrm{TT} v s$. CC: $\mathrm{OR}=2.06,95 \% \mathrm{CI}=1.10-3.84, p=0.021)$ and recessive $(\mathrm{TT}$ vs. $\mathrm{CC}+\mathrm{CT}$ : $\mathrm{OR}=5.93,95 \% \mathrm{CI}=1.42-27.07, p=0.012)$ models (Table 3) was observed. Additionally, a higher frequency of $\mathrm{T}$ allele was detectable in cases compared to controls $(\mathrm{OR}=$ $2.22,95 \% \mathrm{CI}=1.34-3.79, p=0.002)$. A significantly higher frequency of $\mathrm{T}$ allele was also found in males with $\mathrm{AD}$ than in females $(p=0.017)$.

In women, statistical analyses did not reach significance and the risk of $\mathrm{AD}$ was not associated with the studied genetic variations.

Furthermore, binary logistic regression revealed LS and LL genotypes (5-HTTLPR) as independent predictive factors of AD (LS genotype: $\mathrm{OR}=1.81,95 \% \mathrm{CI}=1.07-3.07$, $p=0.027$; LL genotype: $\mathrm{OR}=1.96,95 \% \mathrm{CI}=1.12-3.42, p=$ 0.018 ) (Table 4). In male subgroup of cases, only BDNF TT genotype significantly increased the risk of $\mathrm{AD}(\mathrm{OR}=6.67$, $95 \% \mathrm{CI}=1.39-31.96, p=0.018)$. In females, no significant values were obtained. 
Impact of 5-HTTLPR (ins/del) and BDNF (Val66Met) on the risk of $D D$

The 5-HTTLPR analysis identified a significantly higher frequency of LL genotype or a combination of LL and LS genotypes in patients suffering from DD (UD, BD) than in donors collected as controls (LL vs. SS: reciprocal of OR $=1.80,95 \%$ $\mathrm{CI}=1.01-3.17, p=0.041$, codominant model; LL+LS $v s$. SS: reciprocal of $\mathrm{OR}=1.71,95 \% \mathrm{CI}=1.04-2.82, p=0.037$, recessive model) (Table 2). A similar trend was observed in a separate analysis performed for the risk of UD. However, a significant association was reached only in the codominant model (LL vs. SS: reciprocal of $\mathrm{OR}=1.86,95 \% \mathrm{CI}=1.03-3.36, p=0.034$ ).

When $B D N F$ genetic variation was analyzed, only gender stratified analysis revealed a significant association (Table 3 ). It can be concluded that TT genotype and T allele were more prevalent in male cases with DD than in controls (TT vs. CC: $\mathrm{OR}=6.49,95 \% \mathrm{CI}=1.54-30.69, p=0.012$, codominant model; TT $v s$. CC+CT: OR $=6.10,95 \% \mathrm{CI}=1.49-28.66$, $p=0.024$, recessive model; $\mathrm{T}$ vs. $\mathrm{C}: \mathrm{OR}=1.98,95 \% \mathrm{CI}=$
$1.13-3.45, p=0.016)$. Equally important, the presence of TT genotype or T allele was associated with a significantly higher risk of UD (TT vs. CC: OR = 5.71, 95\% CI $=1.14-27.90, p=$ 0.031, codominant model; TT vs. CC+CT: OR $=5.35,95 \%$ $\mathrm{CI}=1.11-25.94, p=0.035$, recessive model; $\mathrm{T} v s . \mathrm{C}: \mathrm{OR}=$ $1.87,95 \% \mathrm{CI}=1.06-3.39, p=0.033$ ).

The results indicated no statistically significant difference between the tested genetic variations and disorder susceptibility in the subgroup of female participants.

In case of binary logistic regression, only TT (BDNF) genotype was found as an independent risk factor for DD $(\mathrm{OR}=6.10,95 \% \mathrm{CI}=1.23-30.24, p=0.027)$ and for UD $(\mathrm{OR}=5.57,95 \% \mathrm{CI}=1.06-29.31, p=0.043)$ in male subgroup of cases.

\section{Impact of 5-HTTLPR (ins/del) and BDNF (Val66Met) on the risk of $A N X D$}

A preliminary analysis was carried out in the subgroup of patients with ANXDs $(n=58)$. The statistical assessment

Table 4. Binary logistic regression analysis to identify risk genotypes of 5-HTTLPR (ins/del) and BDNF (Val66Met, rs6265) associated with AD susceptibility

\begin{tabular}{|c|c|c|c|c|}
\hline Variable & B & OR & $95 \%$ CI & $p$ value \\
\hline \multicolumn{5}{|l|}{$\mathrm{AD}$ - all patients } \\
\hline 5-HTTLPR (LS genotype) & 0.593 & 1.81 & $1.07-3.07$ & $0.027^{\star}$ \\
\hline 5-HTTLPR (LL genotype) & 0.673 & 1.96 & $1.12-3.42$ & $0.018^{\star}$ \\
\hline BDNF (CT genotype) & 0.029 & 1.03 & $0.69-1.54$ & 0.888 \\
\hline BDNF (TT genotype) & 0.452 & 1.57 & $0.74-3.34$ & 0.241 \\
\hline \multicolumn{5}{|l|}{ AD - males } \\
\hline 5-HTTLPR (LS genotype) & 0.843 & 2.32 & $0.99-5.44$ & 0.052 \\
\hline 5-HTTLPR (LL genotype) & 0.818 & 2.27 & $0.89-5.79$ & 0.088 \\
\hline BDNF (CT genotype) & 0.400 & 1.49 & $0.76-2.92$ & 0.243 \\
\hline BDNF (TT genotype) & 1.898 & 6.67 & $1.39-31.96$ & $0.018^{*}$ \\
\hline \multicolumn{5}{|l|}{ AD - females } \\
\hline 5-HTTLPR (LS genotype) & 0.414 & 1.51 & $0.75-3.05$ & 0.247 \\
\hline 5-HTTLPR (LL genotype) & 0.479 & 1.62 & $0.78-3.34$ & 0.197 \\
\hline BDNF (CT genotype) & -0.202 & 0.82 & $0.49-1.37$ & 0.445 \\
\hline BDNF (TT genotype) & -0.272 & 0.76 & $0.30-1.92$ & 0.565 \\
\hline \multicolumn{5}{|l|}{ DD - all patients } \\
\hline 5-HTTLPR (LS genotype) & 0.414 & 1.51 & $0.88-2.61$ & 0.138 \\
\hline 5-HTTLPR (LL genotype) & 0.477 & 1.61 & $0.90-2.88$ & 0.107 \\
\hline BDNF (CT genotype) & -0.141 & 0.87 & $0.56-1.34$ & 0.524 \\
\hline BDNF (TT genotype) & 0.501 & 1.65 & $0.76-3.59$ & 0.207 \\
\hline \multicolumn{5}{|l|}{ DD - males } \\
\hline 5-HTTLPR (LS genotype) & 0.532 & 1.70 & $0.72-4.06$ & 0.230 \\
\hline 5-HTTLPR (LL genotype) & 0.402 & 1.49 & $0.56-3.99$ & 0.423 \\
\hline BDNF (CT genotype) & 0.166 & 1.18 & $0.56-2.48$ & 0.661 \\
\hline BDNF (TT genotype) & 1.808 & 6.10 & $1.23-30.24$ & $0.027^{\star}$ \\
\hline
\end{tabular}

\begin{tabular}{lrccc}
\hline Variable & $\mathrm{B}$ & $\mathrm{OR}$ & $95 \% \mathrm{CI}$ & $p$ value \\
\hline DD - females & & & & \\
5-HTTLPR (LS genotype) & 0.336 & 1.40 & $0.68-2.89$ & 0.365 \\
5-HTTLPR (LL genotype) & 0.397 & 1.49 & $0.70-3.16$ & 0.302 \\
BDNF (CT genotype) & -0.304 & 0.74 & $0.43-1.27$ & 0.275 \\
BDNF (TT genotype) & -0.151 & 0.86 & $0.34-2.20$ & 0.754 \\
UD - all patients & & & & \\
5-HTTLPR (LS genotype) & 0.366 & 1.44 & $0.82-2.52$ & 0.200 \\
5-HTTLPR (LL genotype) & 0.510 & 1.67 & $0.92-3.00$ & 0.090 \\
BDNF (CT genotype) & -0.109 & 0.90 & $0.58-1.39$ & 0.628 \\
BDNF (TT genotype) & 0.479 & 1.61 & $0.73-3.57$ & 0.238 \\
UD - males & & & & \\
5-HTTLPR (LS genotype) & 0.591 & 1.81 & $0.72-4.55$ & 0.211 \\
5-HTTLPR (LL genotype) & 0.619 & 1.86 & $0.67-5.16$ & 0.235 \\
BDNF (CT genotype) & 0.167 & 1.18 & $0.55-2.56$ & 0.671 \\
BDNF (TT genotype) & 1.717 & 5.57 & $1.06-29.31$ & $0.043^{*}$ \\
UD - females & & & & \\
5-HTTLPR (LS genotype) & 0.252 & 1.29 & $0.62-2.66$ & 0.497 \\
5-HTTLPR (LL genotype) & 0.354 & 1.43 & $0.67-3.03$ & 0.356 \\
BDNF (CT genotype) & -0.262 & 0.77 & $0.44-1.33$ & 0.350 \\
BDNF (TT genotype) & -0.058 & 0.94 & $0.37-2.42$ & 0.903 \\
ANXD - all patients & & & & \\
5-HTTLPR (LS genotype) & 1.479 & 4.39 & $1.27-15.23$ & $0.020^{*}$ \\
5-HTTLPR (LL genotype) & 1.598 & 4.94 & $1.38-17.67$ & $0.014^{*}$ \\
BDNF (CT genotype) & 0.536 & 1.71 & $0.90-3.23$ & 0.099 \\
BDNF (TT genotype) & 0.261 & 1.30 & $0.37-4.51$ & 0.682 \\
\hline
\end{tabular}

$\mathrm{AD}$, affective disorder; ANXD, anxiety disorder; B, binary logistic regression coefficient; CI, confidence interval; DD, depressive disorder; $\mathrm{OR}$, odds ratio; UD, unipolar depression; * significant association. 
showed a significantly increased risk for developing ANXD in cases carrying at least one $\mathrm{L}$ allele in comparison with SS genotype in the codominant (LL vs. SS: reciprocal of OR = $5.07,95 \% \mathrm{CI}=1.55-16.66, p=0.006)$ and recessive $(\mathrm{LL}+\mathrm{LS}$ vs. SS: reciprocal of $\mathrm{OR}=4.71,95 \% \mathrm{CI}=1.53-14.93, p=$ 0.006 ) models (Table 2). There was also a significant association between allele frequency and ANXD risk ( $\mathrm{L} v$ s. S: OR = $1.66,95 \% \mathrm{CI}=1.07-2.57, p=0.020)$ observed. No patient with SS genotype was found among male cases when the gender stratified analysis was performed.

In the case of $B D N F$, our findings indicated only significant associations in males. The CT and TT genotypes (Table 3), as well as $\mathrm{T}$ allele, were positively associated with an increased risk of ANXD in comparison with the reference CC genotype event. reference $\mathrm{C}$ allele (CT vs. $\mathrm{CC}: \mathrm{OR}=3.07$, $95 \% \mathrm{CI}=1.18-8.30, p=0.018$ and TT vs. $\mathrm{CC}: \mathrm{OR}=8.46$, $95 \% \mathrm{CI}=1.52-49.68, p=0.038$, codominant model $\mathrm{CT}+\mathrm{TT}$ vs. $\mathrm{CC}: \mathrm{OR}=3.52,95 \% \mathrm{CI}=1.36-8.87, p=0.005$, dominant model; $\mathrm{T} v s . \mathrm{C}: \mathrm{OR}=2.97,95 \% \mathrm{CI}=1.51-6.04, p=0.002$ ).

Binary logistic regression determined LS and LL genotypes $(\mathrm{OR}=4.39,95 \% \mathrm{CI}=1.27-15.23, p=0.020 ; \mathrm{OR}=4.94$, $95 \% \mathrm{CI}=1.38-17.67, p=0.014$, respectively) as independent predictors of ANXD (Table 4). The gender stratified analysis was not performed due to the small number of cases.

\section{Combined effect of 5-HTTLPR (ins/del) and BDNF (Val66Met) on the risk of $A D$}

Finally, we evaluated the possible combined effects of both tested genetic variations in relation to the development of $\mathrm{ADs}$ and their subtypes. Overall, 9 combinations (LL-CC $=20.0 \%$, $\mathrm{LL}-\mathrm{CT}=9.1 \%, \mathrm{LL}-\mathrm{TT}=1.8 \%, \mathrm{LS}-\mathrm{CC}=32.3 \%, \mathrm{LS}-\mathrm{CT}=13.2 \%$, $\mathrm{LS}-\mathrm{TT}=3.2 \%, \mathrm{SS}-\mathrm{CC}=13.2 \%, \mathrm{SS}-\mathrm{CT}=6.8 \%, \mathrm{SS}-\mathrm{TT}=0.5 \%)$ were found in the control group and 8 combinations (LL-CC $=$ $23.9 \%, \mathrm{LL}-\mathrm{CT}=8.9 \%$, LL-TT $=3.6 \%, \mathrm{LS}-\mathrm{CC}=32.1 \%$, LS-CT $=15.0 \%, \mathrm{LS}-\mathrm{TT}=5.0 \%, \mathrm{SS}-\mathrm{CC}=7.5 \%, \mathrm{SS}-\mathrm{CT}=3.9 \%)$ in the total patient sample. The best model obtained in our study group was the comparison of the distribution of combination LL or LS (5-HTTLPR) and CT or TT (BDNF) among cases and controls (Table 5). A significantly increased risk of developing any type of $\mathrm{AD}$ was observed in male cases carrying this genotype combination in comparison with male controls $(\mathrm{OR}=$ $\left.2.58,95 \% \mathrm{CI}=1.35-4.94, p^{1}=0.004\right)$. A significantly higher frequency of LL or LS and CT or TT combination was also associated with the risk of anxiety susceptibility, including the male subpopulation (all cases with ANXD vs. controls: $\mathrm{OR}=$ 2.02, 95\% CI $=1.11-3.68, p^{2}=0.020$; males with ANXD $v s$. controls: $\left.\mathrm{OR}=4.81,95 \% \mathrm{CI}=1.89-12.19, p^{3}=0.001\right)$. On the other hand, cases carrying a combination of genotypes SS (5-HTTLPR) and TT (BDNF) had a significantly lower chance of developing any type of $\mathrm{AD}(\mathrm{OR}=0.53,95 \% \mathrm{CI}=0.29-0.97$, $\left.p^{4}=0.036\right)$. In the same way, this genotype combination was associated with a lower risk of ANXD $(\mathrm{OR}=0.12,95 \% \mathrm{CI}=$ $\left.0.02-0.87, p^{5}=0.009\right)$. Despite a $p^{6}$-value less than 0.05 , no statistically significant association was found among the subgroup of male cases with ANXD and control males because of values of $95 \% \mathrm{CI}$ outside the required interval.

\section{Discussion}

Our study was aimed to investigate whether two common genetic variations (5-HTTLPR, ins/del and BDNF 196C $>$ T) influence susceptibility to ADs or their subtypes. Moreover, the impact of gene-gene interaction on disorder risk was studied. To our knowledge, this is the first study evaluating these relationships in the Slovakian population sample.

Initially, we observed a higher susceptibility to DDs and ANXDs among females than males, which was in line with previous research (World Health Organization 2017). Next, the L allele (5-HTTLPR) and C allele (BDNF) were found to be the major alleles, the findings being consistent with reports from other European populations (The 1000 Genomes Project Consortium 2015).

Table 5. Analysis of gene-gene interaction of 5-HTTLPR (ins/del) and BDNF (Val66Met, rs6265) between patients and controls

\begin{tabular}{lrrlcr}
\hline & & \multicolumn{4}{c}{ Combination } \\
\cline { 3 - 6 } & & \multicolumn{1}{c}{1} & \multicolumn{1}{c}{2} & 3 & 4 \\
\cline { 3 - 6 } & $N$ & $n(\%)$ & $n(\%)$ & $n(\%)$ & $n(\%)$ \\
\hline Controls & 220 & $115(52.3)$ & $60(27.3)$ & $29(13.2)$ & $16(7.3)$ \\
Males & 86 & $46(53.5)$ & $19(22.1)$ & $16(18.6)$ & $5(5.8)$ \\
Females & 134 & $69(51.5)$ & $41(30.6)$ & $13(9.7)$ & $11(8.2)$ \\
AD & 280 & $157(56.1)$ & $91(32.5)$ & $21(7.5)^{\mathrm{p} 4}$ & $11(3.9)$ \\
Males & 97 & $45(46.4)$ & $41(42.3)^{\mathrm{p} 1}$ & $9(9.3)$ & $2(2.1)$ \\
Females & 183 & $112(61.2)$ & $50(27.3)$ & $12(6.6)$ & $9(4.9)$ \\
DD & 222 & $127(57.2)$ & $66(29.7)$ & $20(9.0)$ & $9(4.1)$ \\
Males & 71 & $34(47.9)$ & $26(36.6)$ & $9(12.7)$ & $2(2.8)$ \\
Females & 151 & $93(61.6)$ & $40(26.5)$ & $11(7.3)$ & $7(4.6)$ \\
UD & 204 & $116(56.9)$ & $61(29.9)$ & $18(8.8)$ & $9(4.4)$ \\
Males & 62 & $31(50.0)$ & $22(35.5)$ & $7(11.3)$ & $2(3.2)$ \\
Females & 142 & $85(59.9)$ & $39(27.5)$ & $11(7.8)$ & $7(4.9)$ \\
ANXD & 58 & $30(51.7)$ & $25(43.1)^{\mathrm{p} 2}$ & $1(1.7)^{\mathrm{p} 5}$ & $2(3.5)$ \\
Males & 26 & $11(42.3)$ & $15(57.7)^{\mathrm{p} 3}$ & $0(0.0)^{\mathrm{p} 6}$ & $0(0.0)$ \\
Females & 32 & $19(59.4)$ & $10(31.3)$ & $1(3.1)$ & $2(6.3)$ \\
\hline
\end{tabular}

$\mathrm{AD}$, affective disorder; $\mathrm{ANXD}$, anxiety disorder; $\mathrm{DD}$, depressive disorder; UD, unipolar depression. $N$, total number of participants; $\mathrm{n}$, the number of participants carrying specific combination of genotypes. Combinations of genotypes: 1: LL or LS (5-HTTLPR) and CC (BDNF); 2: LL or LS (5-HTTLPR) and CT or TT (BDNF); 3: SS (5- HTTLPR) and CC (BDNF); 4: SS (5-HTTLPR) and CT or TT $(B D N F) ;{ }^{\mathrm{p} 1} p=0.004$ ( $\chi^{2}$-test), ${ }^{\mathrm{p} 2} p=0.020$ ( $\chi^{2}$-test), ${ }^{\mathrm{p} 3} p=$ 0.001 ( $\chi^{2}$-test), ${ }^{\mathrm{p} 4} p=0.036$ ( $\chi^{2}$-test), ${ }^{\mathrm{p} 5} p=0.009$ (Fisher's exact test), ${ }^{\mathrm{p} 6} p=0.021$ (Fisher's exact test). 
Regarding 5-HTTLPR (ins/del), the S allele was traditionally considered the risk allele for developing a variety of psychiatric disorders (Lesch et al. 1996). On the contrary, our analysis indicated a significantly increased frequency of LS and LL genotypes in individuals diagnosed with AD than in controls. The subsequent disease-stratified analysis confirmed similar significant associations only in relation to anxiety phenotype. Our results are consistent with findings from several other studies, although these evaluated non-Caucasian populations or nonpathological affective symptomatology. To illustrate, Chinese healthy L carriers had significantly higher anxiety scores than S homozygotes (Long et al. 2013). In addition, Columbian young adults with LL genotype had a higher incidence of anxiety symptoms compared with other 5-HTTLPR genotypes (Jiménez et al. 2019). The $L$ allele was also linked with a higher risk of depression (Peralta-Leal et al. 2012). Another study implicated LL genotype as a predisposing factor for major depression only if individuals had been exposed to negative life events (Zhang et al. 2009). However, a strong relationship between stress events and 5-HTTLPR genotypes contributing to the risk of depression has not been confirmed in a recently published meta-analysis (Culverhouse et al. 2018). Attention was also paid to the evaluation of 5-HTTLPR-affective disorder relationships in specific categories of patients suffering from somatic comorbidities. For example, LL genotype was found to be associated with the mean depression score in patients with coronary artery disease (Meyer et al. 2020). Surprisingly, a recent meta-analysis has identified S allele as a predictive factor for developing depression among Asian and White patients with coronary artery disease (Zhang et al. 2020).

Several mechanisms explaining the consequences of $\mathrm{L}$ allele as a risk factor for ADs have been suggested. For example, the literature showed reduced activation of the amygdala in L homozygotes compared to S carriers (Hariri et al. 2002). Furthermore, reduced amygdala volume and psychopathy scores were presented with the correlations strongest for affective and interpersonal symptoms (Yang et al. 2009). Other findings related to L allele, such as the impact on blood flow in the amygdala, cortisol response to stress, serotonin transport activity, neuropsychological functions were reviewed by Glenn (2011). Moreover, L allele was associated with increased gene transcription, 5-HT transporter level, or its reuptake activity (Lesch et al. 1996), with lower extracellular 5-HT levels expected. Interestingly, a recent animal study has described an association between increased SERT expression in specific brain regions and anxiety-like behavior reversed by the administration of a selective serotonin reuptake inhibitor (Quah et al. 2020). Unexpectedly, this phenomenon was gene independent. Additionally, no relationship between 5-HTTLPR (ins/del) and $\mathrm{AD}$ risk (Mohamed Saini et al. 2012) was reported or
S allele was found as more predictive for disorder development (Miozzo et al. 2020).

Our study also showed a significantly increased frequency of $\mathrm{L}$ allele in male cases compared to male controls, but the result was not confirmed by logistic regression. The possible underlying mechanisms conferring on gender-based associations between genetic variations and diverse psychiatric disorders, including ADs were outlined by some authors (Wang et al. 2014; Zhao et al. 2020). For instance, sex-specific differences in 5-HT signalling (e.g., 5-HT synthesis, the number of nerve cells, 5-HTT gene expression, serotonin transporter degradation), different brain morphology or different responsiveness to environmental factors might have a role in this phenomenon.

In our study, the focus was also on the link between $B D N F$ (rs6265) and the risk of various ADs. Initially, we found a significant deviation from $\mathrm{HWE}$ in the distribution of the $B D N F$ genotypes for the cases. However, in case-control studies, it is recommended to test the deviation from $\mathrm{HWE}$ only in the control group (Ziegler et al. 2010). Additionally, other researchers reported HWE disequilibrium for rs6265 in patients with major depression (Aldoghachi et al. 2019). Furthermore, there was no deviation from HWE for the 5-HTTLPR (ins/del) tested in the same sample of cases in our study. Next, we identified significant associations between rs6265 and disorder risk in gender-stratified analysis. Compared with controls, a significantly higher frequency of TT (MetMet) genotype was found in male cases with any type of AD, as well as with DD and UD. The data obtained are consistent with the hypothesis that $\mathrm{T}$ allele could be implicated in the higher risk of psychiatric disorders due to reduction of trafficking and secretion of BDNF, reduced synaptic transmission, cortical plasticity, and decreased extracellular protein levels (Pattwell et al. 2012). Furthermore, low plasma or serum levels of BDNF were identified in patients suffering from ADs (Aldoghachi et al. 2019; Suliman et al. 2013; Schröter et al. 2020). Conversely, a recent twin study has failed to confirm the relationship between low BDNF levels and DD (Ottesen et al. 2020). Our results are consistent with the prior findings from meta-analysis aimed at the study of rs6265 in patients with major depression (Verhagen et al. 2010). The authors implicated T allele and TT genotype as potential risk factors of the disorder only in males. No significant association was found in the total sample of cases with ADs and females, as well as in ethnicity-stratified analysis. We should mention that a higher frequency of $\mathrm{T}$ allele was identified in male cases compared to females in our study, but this difference reached significance only in the total sample. In contrast to our study, the meta-analysis demonstrated comparable allele distribution among both genders (Verhagen et al. 2010). The gender-related effect of rs6265 on depression risk might be explained by the action of gonadal hormones. 
It has been justified that estrogens have positive regulatory effects on BDNF levels and signalling (Chan and Ye 2017). Thus, we suppose that estrogens can mitigate the negative effect of T allele in females. Overall, sex differences in BDNF signalling and function are elucidated in several articles (Verhagen et al. 2010; Chan and Ye 2017; Tsai 2018). On the other hand, later meta-analyses found no association between rs6265 and major depression (Gyekis et al. 2013) or the effect of the tested genetic variation was moderated by stress events (Zhao et al. 2018). Conversely, a recently published case-control study has demonstrated positive relationships between $\mathrm{T}$ allele and the risk of major depression (Aldoghachi et al. 2019). In the other study, T allele was associated only with the severity of depression (Losenkov et al. 2020). Although an animal study identified a relationship between MetMet genotype and increased risk of anxietyrelated behavior, we found no impact of rs6265 on ANXD susceptibility. As demonstrated in a meta-analysis, BDNF plays an important role mainly in obsessive-compulsive disorder (Suliman et al. 2013). However, specific analyses aimed at subtypes of ANXD were not conducted because of the small sample size. Overall, inconsistent findings from case-control studies evaluating the role of rs6265 in AD development are usually explained by the existence of geneenvironment (Zhao et al. 2018) or gene-gene relationships (Nestor et al. 2019). Next, some studies reported changes in BDNF pathway affected by taking pharmacotherapy (Chen et al. 2010). Other factors have been also outlined, such as the activity of the disorder (acute $v s$. chronic phase), sample size, group differences (e.g., mean age), errors in the selection or implementation of laboratory techniques, even effects of exercise (Ottesen et al. 2020; Szuhany and Otto 2020).

To elucidate the contradictory results from univariate gene association studies, we also examined 5-HTTLPR$B D N F$ combination in predicting the risk of ADs. We found that the probability of a male case with AD to be simultaneously a LL or LS (5-HTTLPR) and CT or TT (BDNF) carrier was significantly higher compared with controls. However, this significant association was subsequently confirmed only in the subgroup of male cases suffering from ANXD. In addition, in the total sample, the combination of SS (5-HTTLRP) and TT (BDNF) was associated with a lower risk of any type of $\mathrm{AD}$, as well as $\mathrm{ANXD}$. In the same line, a recent gene-gene study has pointed out the importance of specific allelic combinations of 5-HTTLPR and BDNF to the risk of depression or anxiety symptoms (Nestor et al. 2019). The combined effect of BDNF-T allele with $S$ allele of 5-HTTLPR showed a protective effect, while the interaction of $\mathrm{T}$ allele with $\mathrm{L}$ allele increased the risk of psychopathology. Moreover, the results were independent of adverse childhood experiences. On the other hand, a prior German analysis supported gene-gene-environment hypothesis.
In case of childhood neglect, participants carrying SS and CC genotypes had the highest risk of DD compared to carriers of $\mathrm{T}$ and $\mathrm{S}$ alleles (Grabe et al. 2012). The L allele increased the risk of the disorder in combination with at least one $\mathrm{T}$ allele. In adolescents, the simultaneous presence of several combinations of 5-HTTLPR and BDNF (SS+CT; $\mathrm{L}$ allele+CC and SS+CC) but not (L allele+TT, SS+TT and $\mathrm{L}$ allele+CT) led to fewer depressive symptoms in case of higher levels of positive parenting (Wang et al. 2020). The importance of the evaluation of gene-gene interactions in relation to $\mathrm{ADs}$ was also supported by other researchers (Kostic et al. 2016).

Overall, we suggest that inconsistent, even contradictory findings in univariate or multivariate analyses of the tested genetic variations in relation to $\mathrm{AD}$ risk could be a consequence of low sample size, different phenotypic heterogeneity of ADs, different classifications of affective disorders, as well as the diversity of the population sample. Moreover, it seems evident that the effects of genetic variations are likely to be much more complex. The more complex pathogenesis of psychiatric disorders is also supported by the fact that the incidence of any type of $\mathrm{AD}$ is lower than the frequency of risk genotypes or alleles.

Although the main strength of our research was that all analyses were performed on the same population sample, several limitations of this study must be underscored. Firstly, the major drawback of the study was the small sample size in the subgroup of patients with ANXD. Thus, our results must be interpreted with caution. Secondly, a lack of analysis of additional genetic variations influencing the functionality of 5-HTTLPR (ins/del) alleles could be another limitation. Hence, mainly genotyping for the nearby genetic variation rs25531 (1936A $>$ G, MAF in European population = 0.026; "triallelic marker" - S, $\mathrm{L}_{\mathrm{A}}$ and $\mathrm{L}_{\mathrm{G}}$ ) located within the $\mathrm{L}$ allele of 5-HTTLPR must be performed in the next larger studies to elucidate the real role of the long allele in psychiatric disorders. Previous research indicated that the $\mathrm{L}_{\mathrm{G}}$ variant functions similarly to the short allele (Praschak-Rieder et al. 2007). However, inconsistent conclusions were observed in gene association studies. Surprisingly, several studies evaluating the Caucasian population revealed that $\mathrm{L}_{\mathrm{A}} \mathrm{L}_{\mathrm{A}}$ carriers can have higher rates of depressive and anxiety disorders or more depressive symptoms (Laucht et al. 2009; Warnke et al. 2020). On the other hand, a meta-analysis evaluating 5-HTTLPR in relation to antidepressant response reported a predictive value of biallelic but not triallelic genetic variation (Ren et al. 2020). Furthermore, a meta-analysis suggested an increasing prevalence of depression over time in the general population (Moreno-Agostino et al. 2021). Thus, a lack of gene-environment interactions could be another limitation. In addition, assessing the impact of hormone levels, concurrent diseases, or receiving pharmacotherapy on the gene-AD relationship could be beneficial. 


\section{Conclusions}

Our findings suggest that both genetic variations 5-HTTLPR (ins/del) and BDNF (rs6265) might have a role in the development of affective disorders. The LS and LL genotypes (5-HT$T L P R$ ) were predictive risk markers rather for ANXD than for $\mathrm{DD}$, while TT $(B D N F)$ genotype mainly increased the risk of $\mathrm{AD}$ in male individuals. However, in gene-gene analysis, the effect of T allele was affected by the 5-HTTLPR background. The LL or LS and CT or TT genotype combinations had a risk-enhancing effect on AD susceptibility (mainly ANXD in males), while SS (5-HTTLPR) and TT (BDNF) combination had a protective effect on AD risk (mainly ANXD). It seems that the tested genetic variations might play more important role in males than in females.

Overall, the translation of lab data into clinical practice represents a great challenge. It has been hypothesized that the understanding the genetic risk factors for affective disorders could help identify risk patients in the early stages, prevent disorder progression, or even use pharmacogenomicsguided interventions of these disorders, which generally may provide a new, more targeting strategy to tackle this serious socio-economic problem. Thus, larger multicenter prospective studies are needed to elucidate the contradictory implications of gene association studies performed in patients suffering from diverse types of affective disorders. Moreover, the evaluation of additional risk factors could be beneficial in such studies to fully understand the role of the tested genetic variations and their combinations.

\section{Conflict of interest. None.}

Acknowledgements. This study was supported by the University Biomedical and Technological park (MediPark, No. ITMS2014:313011D103) and by the Ministry of Health of the Slovak Republic (No. MZ2019/29-UPJŠ-1).

\section{References}

Aldoghachi AF, Tor YS, Redzun SZ, Lokman KAB, Razaq NAA, Shahbudin AF, Badamasi IM, Cheah PS, Stanslas J, Veerakumarasivam A, et al. (2019): Screening of brain-derived neurotrophic factor (BDNF) single nucleotide polymorphisms and plasma BDNF levels among Malaysian major depressive disorder patients. PLoS One 14, e0211241 https://doi.org/10.1371/journal.pone.0211241

Bocchio-Chiavetto L, Bagnardi V, Zanardini R, Molteni R, Nielsen MG, Placentino A, Giovannini C, Rillosi L, Ventriglia M, Riva MA, Gennarelli M (2010): Serum and plasma BDNF levels in major depression: a replication study and meta-analyses. World J. Biol. Psychiatry 11, 763-773 https://doi.org/10.3109/15622971003611319

Border R, Johnson EC, Evans LM, Smolen A, Berley N, Sullivan PF, Keller MC (2019): No support for historical candidate gene or candidate gene-by-interaction hypotheses for major depression across multiple large samples. Am. J. Psychiatry 176, 376-387 https://doi.org/10.1176/appi.ajp.2018.18070881

Chan CB, Ye K (2017): Sex differences in brain-derived neurotrophic factor signaling and functions. J. Neurosci. Res. 95, 328-335. Erratum in (2020): J. Neurosci. Res. 98, 404 https://doi.org/10.1002/jnr.23863

Chen CC, Hsu LW, Huang LT, Huang TL (2010): Chronic administration of cyclosporine A changes expression of BDNF and TrkB in rat hippocampus and midbrain. Neurochem. Res. 35, 1098-1104 https://doi.org/10.1007/s11064-010-0160-0

Culverhouse RC, Saccone NL, Horton A, Ma Y, Anstey KJ, Banaschewski T, Burmeister M, Cohen-Woods S, Etain B, Fisher HL, et al. (2018): Collaborative meta-analysis finds no evidence of a strong interaction between stress and 5-HTTLPR genotyoe contributing to the development of depression. Mol. Psychiatry 23, 133-142

Egan MF, Kojima M, Callicott JH, Goldberg TE, Kolachana BS, Bertolino A, Zaitsev E, Gold B, Goldman D, Dean M, et al. (2003): The BDNF val66met polymorphism affects activity-dependent secretion of BDNF and human memory and hippocampal function. Cell 112, 257-269 https://doi.org/10.1016/S0092-8674(03)00035-7

Glenn AL (2011): The other allele: exploring the long allele of the serotonin transporter gene as a potential risk factor for psychopathy: a review of the parallels in findings. Neurosci. Biobehav. Rev. 35, 612-620 https://doi.org/10.1016/j.neubiorev.2010.07.005

Grabe HJ, Schwahn C, Mahler J, Appel K, Schulz A, Spitzer C, Fenske K, Barnow S, Freyberger HJ, Teumer A, et al. (2012): Genetic epistasis between the brain-derived neurotrophic factor Val66Met polymorphism and the 5-HTT promoter polymorphism moderates the susceptibility to depressive disorders after childhood abuse. Prog. Neuropsychopharmacol. Biol. Psychiatry 36, 264-270 https://doi.org/10.1016/j.pnpbp.2011.09.010

Gyekis JP, Yu W, Dong S, Wang H, Qian J, Kota P, Yang J (2013): No association of genetic variants in BDNF with major depression: a meta- and gene-based analysis. Am. J. Med. Genet. B Neuropsychiatr. Genet. 162B, 61-70 https://doi.org/10.1002/ajmg.b.32122

Hariri AR, Mattay V, Tessitore A, Kolachana B, Fera F, Goldman D, Egan MF, Weinberger DR (2002): Serotonin transporter genetic variation and the response of the human amygdala. Science 297, 400-403

https://doi.org/10.1126/science.1071829

Iurescia S, Seripa D, Rinaldi M (2016): Role of the 5-HTTLPR and SNP promoter polymorphisms on serotonin transporter gene expression: a closer look at genetic architecture and in vitro functional studies of common and uncommon allelic variants. Mol. Neurobiol. 53, 5510-5526 https://doi.org/10.1007/s12035-015-9409-6

Jiménez KM, Pereira-Morales AJ, Adan A, Lopez-Leon S, Forero DA (2019): Anxiety-related endophenotypes and hazardous alcohol use in young adults are associated with a functional polymorphism in the SLC6A4 gene. The Open Neurology Journal 13, 83-91 
https://doi.org/10.2174/1874205X01913010083

Kostic M, Canu E, Agosta F, Munjiza A, Novakovic I, Dobricic V, Maria Ferraro P, Miler Jerkovic V, Pekmezovic T, Lecic Tosevski D, Filippi M (2016): The cumulative effect of genetic polymorphisms on depression and brain structural integrity. Hum. Brain Mapp. 37, 2173-2184

https://doi.org/10.1002/hbm.23165

Laucht M, Treutlein J, Blomeyer D, Buchmann AF, Schmid B, Becker K, Zimmermann US, Schmidt MH, Esser G, Rietschel M, Banaschewski T (2009): Interaction between the 5-HTTLPR serotonin transporter polymorphism and environmental adversity for mood and anxiety psychopathology: evidence from a high-risk community sample of young adults. Int. J. Neuropsychopharmacol. 12, 737-747 https://doi.org/10.1017/S1461145708009875

Lesch KP, Bengel D, Heils A, Sabol SZ, Greenberg BD, Petri S, Benjamin J, Müller CR, Hamer DH, Murphy DL (1996): Association of anxiety-related traits with a polymorphism in the serotonin transporter gene regulatory region. Science 274, 1527-1531 https://doi.org/10.1126/science.274.5292.1527

Lin CC, Huang TL (2020): Brain-derived neurotrophic factor and mental disorders. Biomed. J. 43,134-142 https://doi.org/10.1016/j.bj.2020.01.001

Liu Y, Zhao J, Guo W (2018): Emotional roles of mono-aminergic neurotransmitters in major depressive disorder and anxiety disorders. Front. Psychol. 9, 2201 https://doi.org/10.3389/fpsyg.2018.02201

Long H, Liu B, Hou B, Wang C, Li J, Qin W, Wang D, Zhou Y, Kendrick KM, Yu C, Jiang T (2013): The long rather than the short allele of 5-HTTLPR predisposes Han Chinese to anxiety and reduced connectivity between prefrontal cortex and amygdala. Neurosci. Bull. 29, 4-15 https://doi.org/10.1007/s12264-013-1299-x

Losenkov IS, Mulder NJV, Levchuk LA, Vyalova NM, Loonen AJM, Bosker FJ, Simutkin GG, Boiko AS, Bokhan NA, Wilffert B, et al. (2020): Association between BDNF gene variant rs6265 and the severity of depression in antidepressant treatment-free depressed patients. Front. Psychiatry 11, 38 https://doi.org/10.3389/fpsyt.2020.00038

Martinowich K, Lu B (2008): Interaction between BDNF and serotonin: role in mood disorders. Neuropsychopharmacology $33,73-83$ https://doi.org/10.1038/sj.npp.1301571

Meyer T, Rothe I, Staab J, Deter HC, Fangauf SV, Hamacher S, Hellmich M, Jünger J, Ladwig KH, Michal M, et al. (2020): Length polymorphisms in the angiotensin I-converting enzyme gene and the serotonin-transporter-linked polymorphic region constitute a risk haplotype for depression in patients with coronary artery disease. Biochem. Genet. 58, 631-648 https://doi.org/10.1007/s10528-020-09967-w

Miozzo R, Eaton WW, Joseph Bienvenu O 3rd, Samuels J, Nestadt G (2020): The serotonin transporter gene polymorphism (SLC6A4) and risk for psychiatric morbidity and comorbidity in the Baltimore ECA follow-up study. Compr. Psychiatry 102, 152199 https://doi.org/10.1016/j.comppsych.2020.152199

Mohamed Saini S, Muhamad Radzi A, Abdul Rahman AH (2012): Polymorphism of the serotonin transporter gene (5-HTTLPR) in major depressive disorder patients in Malaysia. Asia Pac. Psychiatry 4,126-130 https://doi.org/10.1111/j.1758-5872.2012.00190.x

Moreno-Agostino D, Wu YT, Daskalopoulou C, Hasan MT, Huisman M, Prina M (2021): Global trends in the prevalence and incidence of depression: a systematic review and meta-analysis. J. Affect Disord. 281, 235-243 https://doi.org/10.1016/j.jad.2020.12.035

Murakami F, Shimomura T, Kotani K, Ikawa S, Nanba E, Adachi K (1999): Anxiety traits associated with a polymorphism in the serotonin transporter gene regulatory region in the Japanese. J. Hum. Genet. 44, 15-17 https://doi.org/10.1007/s100380050098

Nestor PG, O'Donovan K, Lapp HE, Hasler VC, Boodai SB, Hunter $\mathrm{R}$ (2019): Risk and protective effects of serotonin and BDNF genes on stress-related adult psychiatric symptoms. Neurobiol. Stress 11, 100186 https://doi.org/10.1016/j.ynstr.2019.100186

Ottesen NM, Meluken I, Frikke-Schmidt R, Plomgaard P, Scheike T, Kessing LV, Miskowiak K, Vinberg M (2020): S100B and brain derived neurotrophic factor in monozygotic twins with, at risk of and without affective disorders. J. Affect Disord. 274, 726-732 https://doi.org/10.1016/j.jad.2020.05.015

Park H, Poo MM (2013): Neurotrophin regulation of neural circuit development and function. Nat. Rev. Neurosci. 14, 7-23 https://doi.org/10.1038/nrn3379

Pattwell SS, Bath KG, Perez-Castro R, Lee FS, Chao MV, Ninan I (2012): The BDNF Val66Met polymorphism impairs synaptic transmission and plasticity in the infralimbic medial prefrontal cortex. J. Neurosci. 32, 2410-2421 https://doi.org/10.1523/JNEUROSCI.5205-11.2012

Peralta-Leal V, Leal-Ugarte E, Meza-Espinoza JP, Gutiérrez-Angulo M, Hernández-Benítez CT, García-Rodríguez A, DávalosRodríguez IP, Gonzales E, Durán-González J (2012): Association of serotonin transporter gene polymorphism 5-HTTLPR and depressive disorder in a Mexican population. Psychiatr. Genet. 22, 265-266 https://doi.org/10.1097/YPG.0b013e32834f3577

Praschak-Rieder N, Kennedy J, Wilson AA, Hussey D, Boovariwala A, Willeit M, Ginovart N, Tharmalingam S, Masellis M, Houle S, Meyer JH (2007): Novel 5-HTTLPR allele associates with higher serotonin transporter binding in putamen: a [(11)C] DASB positron emission tomography study. Biol. Psychiatry 62, 327-331 https://doi.org/10.1016/j.biopsych.2006.09.022

Quah SKL, McIver L, Roberts AC, Santangelo AM (2020): Trait anxiety mediated by amygdala serotonin transporter in the common marmoset. J. Neurosci. 40, 4739-4749 https://doi.org/10.1523/JNEUROSCI.2930-19.2020

Ren F, Ma Y, Zhu X, Guo R, Wang J, He L (2020): Pharmacogenetic association of bi- and triallelic polymorphisms of SLC6A4 with antidepressant response in major depressive disorder. J. Affect Disord. 273, 254-264 https://doi.org/10.1016/j.jad.2020.04.058

Schröter K, Brum M, Brunkhorst-Kanaan N, Tole F, Ziegler C, Domschke K, Reif A, Kittel-Schneider S (2020): Longitudinal multi-level biomarker analysis of BDNF in major depression 
and bipolar disorder. Eur. Arch. Psychiatry Clin. Neurosci. 270, 169-181 https://doi.org/10.1007/s00406-019-01007-y

Suliman S, Hemmings SM, Seedat S (2013): Brain-Derived Neurotrophic Factor (BDNF) protein levels in anxiety disorders: systematic review and meta-regression analysis. Front. Integr. Neurosci. 7, 55 https://doi.org/10.3389/fnint.2013.00055

Szuhany KL, Otto MW (2020): Assessing BDNF as a mediator of the effects of exercise on depression. J. Psychiatr. Res. 123, 114-118 https://doi.org/10.1016/j.jpsychires.2020.02.003

The 1000 Genomes Project Consortium (2015): A global reference for human genetic variation. Nature 526, 68-74 https://doi.org/10.1038/nature15393

Tsai SJ (2018): Critical issues in BDNF Val66Met genetic studies of neuropsychiatric disorders. Front. Mol. Neurosci. 11, 156 https://doi.org/10.3389/fnmol.2018.00156

Verhagen M, van der Meij A, van Deurzen PA, Janzing JG, AriasVásquez A, Buitelaar JK, Franke B (2010): Meta-analysis of the BDNF Val66Met polymorphism in major depressive disorder: effects of gender and ethnicity. Mol. Psychiatry 15, 260-271 https://doi.org/10.1038/mp.2008.109

Wang M, Tian X, Zhang W (2020): Interactions between the combined genotypes of 5-HTTLPR and BDNF Val66Met polymorphisms and parenting on adolescent depressive symptoms: A three-year longitudinal study. J. Affect Disord. 265, 104-111 https://doi.org/10.1016/j.jad.2020.01.064

Wang TY, Lee SY, Chen SL, Chang YH, Chen SH, Huang SY, Tzeng NS, Wang CL, Yeh PH, Chen KC, et al. (2014): Gender-specific association of the SLC6A4 and DRD2 gene variants in bipolar disorder. Int. J. Neuropsychopharmacol. 17, 211-222 https://doi.org/10.1017/S1461145713001296

Warnke K, Brandt J, Jörgens S, Arolt V, Beer K, Domschke K, Haverkamp W, Kuhlmann SL, Müller-Nordhorn J, Rieckmann N, et al. (2020): Association of 5-HTTLPR/rs25531 with de- pressive symptoms in patients with coronary heart disease: A prospective study. J. Affect Disord. 277, 531-539 https://doi.org/10.1016/j.jad.2020.08.046

World Health Organization (2017): Depression and Other Common Mental Disorders. Global Health Estimates, Geneva

Yang Y, Raine A, Narr KL, Colletti P, Toga AW (2009): Localization of deformations within the amygdala in individuals with psychopathy. Arch. Gen. Psychiatry 66, 986-994 https://doi.org/10.1001/archgenpsychiatry.2009.110

Zhang K, Xu Q, Xu Y, Yang H, Luo J, Sun Y, Sun N, Wang S, Shen Y (2009): The combined effects of the 5-HTTLPR and 5-HTR1A genes modulates the relationship between negative life events and major depressive disorder in a Chinese population. J. Affect Disord. 114, 224-231 https://doi.org/10.1016/j.jad.2008.07.012

Zhang LJ, Zeng XT, Zhao MJ, He DF, Liu JY, Liu MY (2020): The important effect of 5-HTTLPR polymorphism on the risk of depression in patients with coronary heart disease: a metaanalysis. BMC Cardiovasc. Disord. 20, 141 https://doi.org/10.1186/s12872-020-01424-1

Zhao L, Han G, Zhao Y, Jin Y, Ge T, Yang W, Cui R, Xu S, Li B (2020): Gender differences in depression: evidence from genetics. Front. Genet. 11, 562316 https://doi.org/10.3389/fgene.2020.562316

Zhao M, Chen L, Yang J, Han D, Fang D, Qiu X, Yang X, Qiao Z, Ma J, Wang L, et al. (2018): BDNF Val66Met polymorphism, life stress and depression: A meta-analysis of gene-environment interaction. J. Affect Disord. 227, 226-235 https://doi.org/10.1016/j.jad.2017.10.024

Ziegler A, van Steen K, Wellek S (2010): Investigating HardyWeinberg equilibrium in case-control or cohort studies or meta-analysis. Breast Cancer Res. Treat. 128, 197-201 https://doi.org/10.1007/s10549-010-1295-Z

Received: May 21, 2021

Final version accepted: July 14, 2021 